\title{
CURVA DE RETENÇÃo de ÁGUA NO SOLO PELO MÉTODO DO PAPEL-FILTRO ${ }^{(1)}$
}

\author{
Juliana Fenner Ruas Lucas ${ }^{(2)}$, Maria Hermínia Ferreira Tavares $^{(3)}$, Décio \\ Lopes Cardoso $^{(4)}$ \& Fabio Augusto Meira Cássaro ${ }^{(5)}$
}

\begin{abstract}
RESUMO
O método mais tradicional para determinação da curva de retenção de água no solo emprega a câmara de pressão de Richards. Por questões práticas, a busca por métodos alternativos ao de Richards, financeiramente mais acessíveis e menos morosos, faz-se interessante. Diante disso, desenvolveu-se o presente trabalho com o objetivo de avaliar o uso do método do papel-filtro. Para isso, realizou-se inicialmente coleta, caracterização física e preparo de amostras indeformadas de um Latossolo Vermelho distroférrico típico e ensaio em câmara de Richards, utilizando pressões de $0,10,30,60,100,300,500,1000$ e $1500 \mathrm{kPa}$. No ensaio com o papel-filtro, mediu-se o potencial matricial da água em amostras cujas umidades foram previamente estabelecidas, utilizando-se curva de calibração adequada. Os ensaios resultaram em pontos de pressão versus umidade, que foram ajustados pelo modelo de van Genuchten, utilizando o programa RETC. Realizou-se uma análise comparativa de valores de umidade volumétrica estimados pelo modelo ajustado nos ensaios com o papel-filtro com a curva de retenção ajustada obtida pela câmara de Richards. Por meio dessa comparação, verificou-se a aplicabilidade do método do papel-filtro para determinação da curva de retenção de água no solo agrícola utilizado.
\end{abstract}

Termos de indexação: câmara de pressão de Richards, método alternativo, potencial matricial, umidade do solo.

\footnotetext{
(1) Parte da Dissertação de Mestrado da primeira autora junto ao Programa de Pós-Graduação em Engenharia Agrícola da Universidade Estadual do Oeste do Paraná - UNIOESTE. Recebido para publicação em 12 de outubro de 2010 e aprovado em 27 de setembro de 2011.

(2) Mestre em Engenharia Agrícola, Laboratório de Materiais, Universidade Estadual do Oeste do Paraná - UNIOESTE. Av. Tarquínio Joslin dos Santos 1300, CEP 85870-900, Foz do Iguaçu (PR). E-mail: juliana_fenner@hotmail.com

(3) Professora Doutora, Programa de Pós-Graduação em Engenharia Agrícola, Universidade Estadual do Oeste do Paraná UNIOESTE. Rua Universitária 2069, CEP 85870-650, Cascavel (PR). E-mail: mhstavar@gmail.com

(4) Professor Doutor, Centro de Ciências Exatas e Tecnológicas, Universidade Estadual do Oeste do Paraná - UNIOESTE. Rua Universitária 2069, CEP 85819-110, Cascavel (PR). E-mail: deciolc@gmail.com

(5) Professor Doutor, Departamento de Física, Universidade Estadual de Ponta Grossa - UEPG. Av. General Carlos Cavalcanti 4748, CEP 84030-900, Uvaranas (PR). E-mail: fcassaro@uepg.br
} 


\title{
SUMMARY: SOIL-WATER RETENTION CURVE BY THE FILTER PAPER METHOD
}

\begin{abstract}
The most traditional method to determine the soil-water retention curve is based on the Richards' pressure plate apparatus. For practical reasons, cheaper and faster alternatives to the Richards'apparatus are needed. Therefore, this study evaluated the use of the filter paper method. Initially, an Oxisol was sampled and physically characterized. Undisturbed soil samples were prepared and tested in Richards'chamber, at pressures of 0, 10, 30, 60, 100, 300, $500,1,000$ and 1,500 $\mathrm{kPa}$. In the filter paper test, the water matric potential was measured in samples for which moisture levels had been determined by an appropriate calibration curve. The tests obtained pressure versus moisture points that were adjusted by the van Genuchten model, using the RETC program. A comparative analysis of the soil moisture values estimated by the model adjusted in the filter paper test and the adjusted retention curve obtained by the Richards' apparatus was performed. Results allowed the conclusion that the filter paper method is suited to determine the water retention curve of the agricultural soil studied.
\end{abstract}

Index terms: Richards'pressure apparatus, alternative method, matric potential, soil moisture.

\section{INTRODUÇÃO}

A curva de retenção de água no solo tem sido utilizada como importante ferramenta na descrição do comportamento físico-hídrico e na mecânica dos solos não saturados. A curva é parte fundamental da caracterização das propriedades hidráulicas do solo (Cichota \& Jong van Lier, 2004), especialmente em estudos de balanço e disponibilidade de água às plantas, de dinâmica da água e solutos no solo, de infiltração e no manejo da irrigação (Tormena \& Silva, 2002).

A exemplo da distribuição do tamanho dos poros no solo (Cássaro et al., 2008), é possível obter relações de dependência entre os coeficientes das equações matemáticas que descrevem a curva de retenção e diversas propriedades do solo. Mediante essas interrelações, a curva de retenção pode ser utilizada na avaliação da qualidade do solo, indicando práticas mais adequadas de produção agrícola (Machado et al., 2008), assim como no que concerne à estabilidade de obras de terra (Diene \& Mahler, 2007).

Para obtenção da curva de retenção, tradicionalmente utiliza-se a câmara de pressão de Richards. Esse método, entretanto, apresenta algumas dificuldades: a determinação do ponto de equilíbrio entre a pressão aplicada e a água retida no solo, o contato amostra-placa, o longo tempo exigido para as medidas e o custo do equipamento (Tavares et al., 2008).

Por questões práticas, métodos alternativos à câmara de Richards vêm sendo pesquisados. Um exemplo é apresentado por Nascimento et al. (2010), que avaliaram o uso do método de Arya \& Paris (1981) em amostras de um Neossolo Quartzarênico. Esse método fundamenta-se na similaridade entre as funções que descrevem a distribuição de tamanho de partículas do solo e a curva de retenção, empregando um programa computacional baseado no modelo de Arya \& Paris (1981) associado a um analisador granulométrico automático.

Com o objetivo de identificar uma alternativa metodológica, financeiramente mais acessível e menos morosa que a câmara de pressão de Richards, avaliouse a utilização do método do papel-filtro, empregado na medição do potencial matricial da água em amostras de solo, para obtenção da curva de retenção de água em um solo agrícola.

\section{MÉTODO DO PAPEL-FILTRO}

$\mathrm{O}$ uso do papel-filtro para medir o potencial matricial da água no solo baseia-se no princípio de que, quando solo úmido é colocado em contato com um material poroso que possua capacidade de adsorver água, esta será transferida do solo para esse material até que o equilíbrio seja alcançado. No estado de equilíbrio, os potenciais matriciais da água no solo e no material poroso igualam-se, apesar de as respectivas umidades serem diferentes (Marinho \& Oliveira, 2006).

$\mathrm{O}$ método consiste em colocar um papel-filtro em contato com o solo, em um recipiente hermeticamente fechado, por um período de tempo suficiente para que a água nos poros da amostra, nos poros do papel-filtro e no ar no interior do recipiente atinja o equilíbrio. A massa do papel-filtro é posteriormente determinada e o potencial matricial na amostra é inferido a partir de uma curva de calibração do papel, a qual relaciona o potencial e o teor de água neste (ASTM, 2003).

Uma vez que o método do papel-filtro determina o potencial matricial do solo de forma indireta, sua 
precisão depende da precisão com que foi determinada a curva de calibração. Outros aspectos que influenciam na confiabilidade das medições são: variação de temperatura, tempo de equilíbrio, contato entre papel-filtro e amostra, manuseio e exposição ao ambiente (Bicalho et al., 2007).

A flutuação de temperatura durante o período de equilíbrio aumenta a variabilidade dos resultados (AlKhafaf \& Hanks, 1974). Assim, é imprescindível minimizar os gradientes de temperatura durante a medição do potencial matricial, bem como durante a calibração com soluções salinas (Bulut \& Leong, 2008). Considerando uma temperatura nominal de $20^{\circ} \mathrm{C}$, a variação de temperatura deve ser menor que $3{ }^{\circ} \mathrm{C}$ (ASTM, 2003).

O tempo é fator importante no método do papelfiltro, pois deve ser suficiente para que o equilíbrio seja alcançado (Marinho \& Oliveira, 2006). O período de sete dias é considerado suficiente para garantir o equilíbrio na maioria das medições de potencial matricial (Leong et al., 2002), embora períodos maiores possam ser necessários para potenciais muito baixos (Fawcett \& Collis-George, 1967). Para a calibração do papel-filtro com soluções salinas, na literatura recente é sugerido um período de 14 dias (Bulut \& Wray, 2005).

Gomes (2007) adverte que irregularidades superficiais ou descontinuidades na face da amostra em relação ao papel-filtro podem alterar significativamente a precisão das aferições, acarretando medições incorretas em razão da deficiência de contato entre o papelfiltro e o solo. Entretanto, esse autor salienta também que o aumento do tempo de interação entre o papel-filtro e o solo pode compensar eventuais dificuldades de acesso da água intersticial ao papel-filtro.

Para obter-se uma medição confiável de potencial matricial, é necessário garantir que o papel-filtro, após o equilíbrio, seja removido do ambiente de equilíbrio sem alteração significativa de umidade. Por esse motivo, o manuseio deve ser o mais rápido possível, até ser aferida a quantidade de água no papel-filtro (Bulut et al., 2001).

O método do papel-filtro é padronizado pela norma D5298-03 (ASTM, 2003), que delineia a calibração do papel-filtro e o procedimento de medição.

\section{MATERIAL E MÉTODOS}

\section{Coleta de solo e confecção de amostras}

O estudo foi conduzido em amostras preparadas a partir de solo coletado no Núcleo Experimental de Engenharia Agrícola, pertencente à Universidade Estadual do Oeste do Paraná, localizado na BR 467, $\mathrm{km}$ 101, na cidade de Cascavel, com coordenadas geográficas de $24^{\circ} 54^{\prime} 148^{\prime \prime}$ latitude sul, $53^{\circ} 31^{\prime}$ 57.312" longitude oeste e altitude de $750 \mathrm{~m}$.
O solo utilizado no trabalho é classificado como Latossolo Vermelho distroférrico típico. Os atributos físicos determinados para esse solo foram: umidade gravimétrica de coleta $(\mathrm{ABNT}, 1986)=0,39 \mathrm{~g} \mathrm{~g}^{-1}$; densidade de partícula $(\mathrm{ABNT}, 1984)=2,95 \mathrm{~g} \mathrm{~cm}^{-3}$; densidade do solo (método do anel volumétrico (Embrapa, 2005)) $=1,09 \mathrm{~g} \mathrm{~cm}^{-3} ;$ e análise granulométrica (método da pipeta (Embrapa, 2005)) $=750 \mathrm{~g} \mathrm{~kg}^{-1}$ de argila, $130 \mathrm{~g} \mathrm{~kg}^{-1}$ de silte e $120 \mathrm{~g} \mathrm{~kg}^{-1}$ de areia.

A extração de blocos de solo com estrutura indeformada foi feita utilizando cilindros de plástico de $0,12 \mathrm{~m}$ de altura e $0,10 \mathrm{~m}$ de diâmetro. $\mathrm{O}$ solo foi retirado de uma área de $1,5 \mathrm{~m}$ de diâmetro, a uma profundidade de 0,28-0,40 m da superfície, para evitar a presença de raízes, em local agricultável, embora coberto por pastagem na ocasião da coleta.

A partir dos blocos cilíndricos de solo, foram esculpidas amostras indeformadas cilíndricas de 0,05 $\mathrm{m}$ de diâmetro e 0,05 $\mathrm{m}$ de altura para os ensaios na câmara de pressão de Richards e de aproximadamente $0,05 \mathrm{~m}$ de diâmetro e 0,025 $\mathrm{m}$ de altura para os ensaios com o papel-filtro. À medida que os blocos de solo eram desbastados até atingirem o diâmetro definido, cilindros plásticos rígidos eram encaixados nas amostras para possibilitar o processo de saturação destas. Uma vez confeccionadas as amostras, efetuaram-se medidas de diâmetro e de altura, com paquímetro, para determinar o volume das mesmas.

\section{Ensaio com a câmara de pressão de Richards}

Cinco das amostras indeformadas, com as características anteriormente descritas, foram submetidas ao ensaio com a câmara de pressão de Richards (Embrapa, 2005), sendo utilizadas as pressões de $0,10,30,60,100,300,500,1000$ e $1500 \mathrm{kPa}$. Este ensaio foi realizado pelo Laboratório de Física do Solo do Departamento de Ciência do Solo da ESALQ Universidade de São Paulo, campus de Piracicaba, demandando aproximadamente 60 dias para a sua conclusão.

\section{Ensaio com o papel-filtro}

A execução do ensaio com o papel-filtro foi realizada com base na norma D5298-03 (ASTM, 2003), empregando-se papel-filtro Whatman Schleicher \& Schuell 42. Para cada medida de potencial matricial, foram utilizadas duas amostras de solo, com as características anteriormente descritas. Para obtenção da curva de retenção de água no solo aplicando o método do papel-filtro, prepararam-se 40 pares de amostras com diferentes umidades.

Para cada par de amostras, procedeu-se à montagem indicada pela norma D5298-03, sendo disposto um papel-filtro de $0,045 \mathrm{~m}$ de diâmetro entre dois papéis-filtro de $0,05 \mathrm{~m}$, e estes, colocados entre duas amostras de solo com igual umidade, dentro de 
um recipiente plástico com tampa. Este recipiente foi fechado e acondicionado em ambiente climatizado, onde as temperaturas máxima e mínima registradas foram de 22,2 e $19,5^{\circ} \mathrm{C}$, respectivamente, por um período de 30 dias, para garantir o equilíbrio termodinâmico da água no sistema solo/papel-filtro.

Ao fim do período de equilíbrio, realizaram-se as mensurações de massa necessárias do papel-filtro central de cada montagem, de modo a determinar-se o teor de água, $\mathrm{w}_{\mathrm{f}}$, neste. Nesse procedimento, o manuseio do papel-filtro de cada montagem ocorreu em até $5 \mathrm{~s}$. Ao término do ensaio, após a manipulação dos papéis-filtro, as amostras de solo foram secas em estufa a $105^{\circ} \mathrm{C}$, por $24 \mathrm{~h}$, e tiveram suas massas aferidas, para determinação da umidade volumétrica destas.

\section{Calibração do papel-filtro}

A curva de calibração do papel-filtro foi obtida empregando-se soluções salinas, com base no que determina a norma D5298-03 (ASTM, 2003) e nas orientações de Bulut et al. (2001), que detalham esse procedimento. Utilizaram-se os valores tabelados de concentração de solução de $\mathrm{KCl}$ versus sucção produzida por esta, a $20{ }^{\circ} \mathrm{C}$, apresentados por Bulut (1996) e Fredlund \& Rahardjo (1993), para definir as concentrações das soluções a serem empregadas no processo.

Foram preparadas 14 soluções de $\mathrm{KCl}$ nas concentrações de 0,$001 ; 0,002 ; 0,005 ; 0,010 ; 0,050$; 0,$100 ; 0,150 ; 0,200 ; 0,250 ; 0,300 ; 0,350 ; 0,400 ; 0,450$; e $0,500 \mathrm{~mol} \mathrm{~kg}^{-1}$. Cada solução foi colocada em um recipiente plástico com tampa, juntamente com um suporte plástico para apoiar um papel-filtro seco a 0,02 $\mathrm{m}$ acima da superfície da solução. Feita essa montagem, os recipientes com as soluções salinas foram fechados e acondicionados em ambiente com temperatura controlada de $20{ }^{\circ} \mathrm{C}$, por 30 dias, para garantir o equilíbrio termodinâmico da água dentro deles.

Ao fim do período de equilíbrio, procedeu-se à determinação do teor de água no papel-filtro de cada montagem, da mesma forma já empregada com os papéis-filtro centrais do ensaio com as amostras de solo, obtendo-se os valores de $\mathrm{w}_{\mathrm{f}}$ referentes a cada solução salina. Efetuando regressão linear nos valores de sucção e teor de água no papel-filtro, obteve-se a curva de calibração do papel-filtro utilizado nos ensaios:

$$
\log \psi_{\mathrm{m}}=4,9322-0,0836 \mathrm{w}_{\mathrm{f}}
$$

em que $\psi_{\mathrm{m}}$ é a sucção ou potencial matricial, em $\mathrm{kPa}$. $\mathrm{O}$ coeficiente de determinação $\mathrm{R}^{2}$ fornecido pelo programa para a equação resultou em 99,25\%. Aplicando-se na equação 1 os valores de $\mathrm{w}_{\mathrm{f}}$ dos papéisfiltro centrais da montagem com as amostras de solo submetidas ao ensaio, obteve-se o potencial matricial da água nestas.

\section{Análise dos resultados}

Aplicou-se estatística descritiva para analisar os resultados de umidade volumétrica obtidos a cada pressão aplicada nos ensaios com a câmara de Richards.

Para efetuar o ajuste dos resultados de umidade volumétrica, $\theta$, versus potencial matricial, $\psi_{\mathrm{m}}$, fornecidos pela câmara de Richards e pelo método do papel-filtro, foram utilizados o modelo de van Genuchten (1980) e o programa RETC (van Genuchten et al., 2009). Identificou-se a configuração - entre as disponíveis no programa - que apresentasse o maior valor para o coeficiente de determinação, $R^{2}$, para o conjunto de dados observados. Esse foi considerado o melhor ajuste possível dos dados.

A fim de verificar a aplicabilidade do papel-filtro para determinação da curva de retenção de água no solo, compararam-se os valores estimados de $\theta$ para $10,30,60,100,300,500,1000,1500 \mathrm{kPa}$ pelas equações fornecidas pelo RETC (van Genuchten et al., 2009) com os valores estimados para a câmara de pressão de Richards para as mesmas pressões, considerando estes como referência.

\section{RESULTADOS}

Na figura 1 são apresentados os valores da umidade volumétrica e do potencial matricial obtidos nos ensaios com a câmara de pressão de Richards e com o papelfiltro.

A estatística descritiva dos valores de $\theta$ em função das pressões aplicadas (potenciais matriciais, $\psi_{\mathrm{m}}$ ) com a câmara de Richards é apresentada no quadro 1.

Observa-se (Quadro 1) que os coeficientes de variação, $\mathrm{CV}$, das umidades volumétricas para as

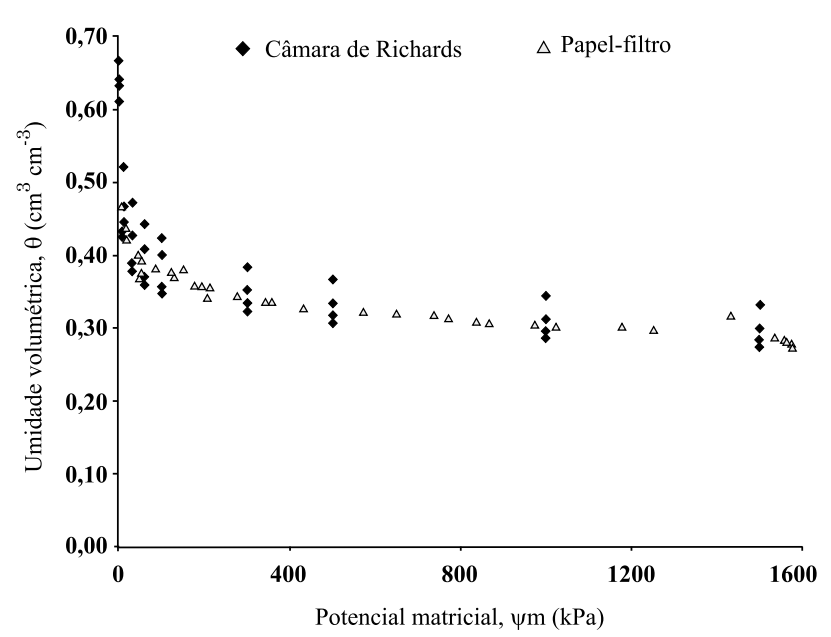

Figura 1. Valores de unidade volumétrica e potencial matricial obtidos com a câmara de pressão de Richards e com o papel-filtro. 


\section{Quadro 1. Estatística descritiva dos valores de umidade volumétrica obtidos na câmara de Richards}

\begin{tabular}{lccccccc}
\hline \multicolumn{1}{c}{ Variável } & N & Média & Desvio-padrão & Variância & Coeficiente de variação & Mínimo & Máximo \\
\hline$\theta(0 \mathrm{kPa})$ & 5 & 0,6368 & 0,0198 & 0,00039 & 3,12 & 0,6105 & 0,6658 \\
$\theta(10 \mathrm{kPa})$ & 5 & 0,4586 & 0,0382 & 0,00146 & 8,32 & 0,4301 & 0,5210 \\
$\theta(30 \mathrm{kPa})$ & 5 & 0,4084 & 0,0413 & 0,00170 & 10,11 & 0,3770 & 0,4720 \\
$\theta(60 \mathrm{kPa})$ & 5 & 0,3880 & 0,0372 & 0,00138 & 9,58 & 0,3592 & 0,4434 \\
$\theta(100 \mathrm{kPa})$ & 5 & 0,3753 & 0,0346 & 0,00120 & 9,22 & 0,3482 & 0,4235 \\
$\theta(300 \mathrm{kPa})$ & 5 & 0,3435 & 0,0252 & 0,00064 & 7,34 & 0,3239 & 0,3836 \\
$\theta(500 \mathrm{kPa})$ & 5 & 0,3268 & 0,0247 & 0,00061 & 7,56 & 0,3078 & 0,3664 \\
$\theta(1000 \mathrm{kPa})$ & 5 & 0,3054 & 0,0240 & 0,00058 & 0,2872 & 0,3442 \\
$\theta(1500 \mathrm{kPa})$ & 5 & 0,2935 & 0,0236 & 0,00056 & 0,03 & 0,2758 & 0,3319 \\
\hline
\end{tabular}

pressões do ensaio são inferiores a $10 \%$, exceto para a pressão de $30 \mathrm{kPa}(\mathrm{CV}=10,11 \%)$. Segundo PimentelGomes \& Garcia (2002), esses resultados são homogêneos, indicando que os ensaios foram executados em condições bem controladas. $\mathrm{O}$ comportamento dos dados está em concordância com os resultados obtidos por Moraes et al. (1993), que, após analisarem 250 curvas de retenção de água no solo, verificaram que as umidades, nas várias pressões consideradas em seus experimentos, apresentaram, comparativamente, coeficientes de variação baixos, menores que $10 \%$.

Nos ensaios realizados com o método do papel-filtro - uma vez que foram preparados 40 pares de amostras com umidades volumétricas diferentes e, dessa forma, não obtiveram-se repetições para uma mesma umidade - não foi possível efetuar a mesma análise estatística aplicada aos resultados da câmara de Richards.

Calculando o coeficiente de correlação de Pearson para os valores de $\theta$ das amostras e o teor de água no papel-filtro, $\mathrm{w}_{\mathrm{f}}$, obteve-se um valor de 0,961, em um nível de significância de $5 \%$, o que indica forte correlação entre as respectivas medidas.

Para os valores obtidos no ensaio na câmara de Richards, o melhor ajuste dos 45 valores de $\theta$ versus $\psi_{\mathrm{m}}$ é apresentado na equação 2 :

$$
\theta=0,01778+\frac{0,61906}{\left[1+\left(0,44276 \cdot \psi_{m}\right)^{1,09074}\right]^{0,08319}}
$$

em que $\theta$ é dado em $\mathrm{cm}^{3} \mathrm{~cm}^{-3}$ e $\psi_{\mathrm{m}}$ em $\mathrm{cm} \mathrm{H}_{2} \mathrm{O}$, considerando que o programa forneceu os seguintes parâmetros: $\theta_{\mathrm{r}}=0,01778 \mathrm{~cm}^{3} \mathrm{~cm}^{-3} ; \theta_{\mathrm{s}}=0,63684 \mathrm{~cm}^{3} \mathrm{~cm}^{-3}$; $\alpha=0,44276 ; \mathrm{m}=0,08319 ; \mathrm{e} \mathrm{n}=1,09074$, sendo que $\theta_{\mathrm{r}}$ e $\theta_{\mathrm{s}}$ indicam os valores de umidade residual e umidade na saturação, respectivamente, e $\alpha, m$ e $n$ são parâmetros empíricos que devem ser estimados pelo ajuste dos dados. A equação 2 apresentou coeficiente de determinação, $\mathrm{R}^{2}$, de 0,9279 (Hines et al., 2006), em um nível de significância de $5 \%$, indicando cor- respondência adequada entre os valores obtidos e os estimados pelo ajuste.

Quanto aos valores obtidos no ensaio com o papelfiltro, o melhor ajuste dos 40 valores de $\theta$ versus $\psi_{\mathrm{m}}$ é apresentado na equação 3 :

$$
\theta=0,12062+\frac{0,42784}{\left[1+\left(0,05219 \cdot \psi_{\mathrm{m}}\right)^{1,00500}\right]^{0,13404}}
$$

em que $\theta$ é dado em $\mathrm{cm}^{3} \mathrm{~cm}^{-3}$ e $\psi_{\mathrm{m}}$ em $\mathrm{cm} \mathrm{H}_{2} \mathrm{O}$, considerando que o programa forneceu os seguintes parâmetros: $\theta_{\mathrm{r}}=0,12062 \mathrm{~cm}^{3} \mathrm{~cm}^{-3} ; \theta_{\mathrm{s}}=0,54846 \mathrm{~cm}^{3} \mathrm{~cm}^{-3}$; $\alpha=0,05219 ; \mathrm{m}=0,13404 ; \mathrm{e} n=1,00500$. A equação 3 apresentou $\mathrm{R}^{2}=95,44 \%$, em um nível de significância de $5 \%$, indicando elevada eficiência do ajuste dos dados pelo modelo.

A comparação entre os valores estimados de $\theta$ para a câmara de pressão de Richards e para o método do papel-filtro, nas pressões de ensaio da câmara de Richards, é apresentada na figura 2.

Verifica-se (Figura 2) que os valores de $\theta$ estimados para os dois métodos foram bastante semelhantes, o que evidencia a possibilidade de utilização do método do papel-filtro na construção da curva de retenção de água para um solo agrícola. Em parte, esse comportamento deve-se ao ajuste realizado pelo programa RETC (van Genuchten et al., 2009), que, embora fornecendo parâmetros distintos para o modelo de van Genuchten (1980), resultou em respostas convergentes.

Nascimento et al. (2010) não identificaram correspondência entre os valores de umidade do solo estimados pela curva de retenção obtida pelo método de Arya \& Paris (1981) e pela câmara de Richards, sendo aqueles superiores aos valores de umidade determinados pelo método tradicional, na mesma pressão. Esses autores atribuem o resultado à necessidade de desenvolvimento de calibrações específicas do método para o solo estudado necessidade que não ocorre com o método do papelfiltro, cuja calibração independe do tipo de solo. 


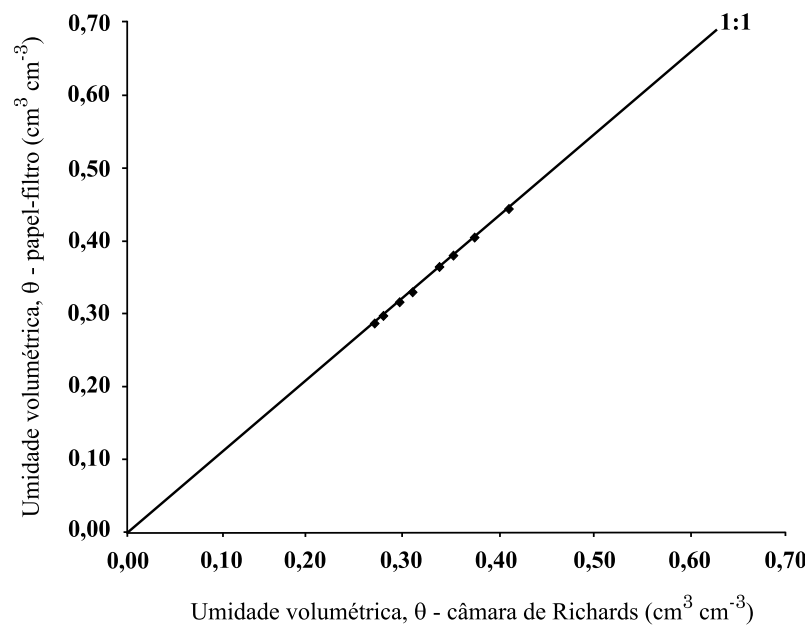

Figura 2. Valores da umidade volumétrica estimada pela câmara de Richards e pelo método do papelfiltro.

Possivelmente, as correlações positivas resultam do rigor imposto aos ensaios realizados e ao preparo de amostras. Destaca-se que - entre os aspectos que influenciam a confiabilidade das medições de $\psi_{\mathrm{m}}$ com o papel-filtro, citados por Bicalho et al. (2007) - a variação de temperatura durante o período de equilíbrio e o tempo de exposição ao ambiente atenderam às recomendações da norma D5298-03 (ASTM, 2003). Já o tempo de equilíbrio foi propositalmente aumentado, podendo ter compensado a interferência de eventuais descontinuidades na face da amostra em relação ao papel-filtro, conforme indicado por Gomes (2007).

De acordo com Bulut \& Leong (2008), o método do papel-filtro é uma técnica simples e confiável para a medição do potencial matricial, desde que seus princípios básicos sejam corretamente compreendidos e um protocolo laboratorial seja cuidadosamente seguido. A exemplo dos trabalhos de Pereira (2004) e Soto (2004), o método também tem sido utilizado para determinar a curva de retenção de água em amostras de solo compactado.

Pelos resultados obtidos, verificou-se que o método do papel-filtro pode ser utilizado para obtenção da curva de retenção de água em um Latossolo Vermelho distroférrico típico, agricultável, com atributos físicos semelhantes aos identificados no solo usado no presente trabalho, constituindo o seu uso em alternativa metodológica à câmara de pressão de Richards.

\section{AGRADECIMENTOS}

Ao Programa de Pós-Graduação em Engenharia Agrícola da Universidade Estadual do Oeste do Paraná, pelo auxílio financeiro para a realização dos ensaios.

\section{LITERATURA CITADA}

ARYA, L.M. \& PARIS, J.F. A physicoempirical model to predict soil moisture characteristics from particle-size distribution and bulk density data. Soil Sci. Soc. Am. J., 45:1023-1030, 1981.

AL-KHAFAF, S. \& HANKS, R.J. Evaluation of the filter paper method for estimating soil water potential. Soil Sci., 117:194-199, 1974.

AMERICAN SOCIETY FOR TESTING AND MATERIALS ASTM. D5298-03: Standard test method for measurement of soil potential (suction) using filter paper. Philadelphia, 2003. $6 \mathrm{p}$.

ASSOCIAC̃̃̃O BRASILEIRA DE NORMAS TÉCNICAS ABNT. NBR 6457: Amostras de solo: preparação para ensaios de compactação e ensaios de caracterização. Rio de Janeiro, 1986. 9p

ASSOCIAÇÃO BRASILEIRA DE NORMAS TÉCNICAS ABNT. NBR 6508: Grãos de solos que passam na peneira de 4,8 mm: determinação da massa específica. Rio de Janeiro, 1984. 8p.

BICALHO, K.V.; CORREIA, A.G.; FERREIRA, S.; FLEUREAU, J.M. \& MARINHO, F.A.M. The use of filter paper method for matric suction measurement of an unsaturated compacted silty sand. In: SIMPÓSIO BRASILEIRO DE SOLOS NÃO SATURADOS, 6., Salvador, 2007. Anais... Salvador, Associação Brasileira de Mecânica dos Solos e Engenharia Geotécnica, 2007. p.215-219.

BULUT, R. A re-evaluation of the filter paper method of measuring soil suction. Lubbock, Texas Tech University, 1996. 89p. (Tese de Mestrado)

BULUT, R. \& LEONG, E.C. Indirect measurement of suction. Geotech. Geol. Eng., 26:633-644, 2008.

BULUT, R.; LYTTON, R.L. \& WRAY, W.K. Soil suction measurements by filter paper. In: GEO-INSTITUTE SHALLOW FOUNDATION AND SOIL PROPERTIES, Houston, 2001. Proceedings... Houston, Geo-Institute of the American Society of Civil Engineers, 2001. p.243-261.

BULUT, R. \& WRAY, W.K. Free energy of water-suction-in filter papers. Geotech. Test. J., 28:355-364, 2005.

CÁSSARO, F.A.M.; PIRES, L.F.; SANTOS, R.A.; GIMÉNEZ, D. \& REICHARDT, K. Funil de Haines modificado: curvas de retenção de solos próximos à saturação. R. Bras. Ci. Solo, 32:2555-2562, 2008

CICHOTA, R. \& JONG van LIER, Q. Análise da variabilidade espacial de pontos amostrais da curva de retenção de água no solo. R. Bras. Ci. Solo, 28:585-596, 2004.

DIENE, A.A. \& MAHLER, C.F. Um instrumento para medida de potencial matricial nos solos sem ocorrência de cavitação. R. Bras. Ci. Solo, 31:1261-1270, 2007.

EMPRESA BRASILEIRA DE PESQUISA AGROPECUÁRIA EMBRAPA. Manual de laboratórios: Solo, água, nutrição vegetal, nutrição animal e alimentos. São Carlos, Embrapa Pecuária Sudeste, 2005. 334p. 
FAWCETT, R.G. \& COLLIS-GEORGE, N. A filter-paper method for determining the moisture characteristics of soil. Austr. J. Exper. Agric. Anim. Husb., 7:162-167, 1967.

FREDLUND, D.G. \& RAHARDJO, H. Soil mechanics for unsaturated soils. Hoboken, John Wiley \& Sons, 1993. 489 p.

GOMES, J.E.S. Estudo sobre os fatores que influenciam a acurácia do método do papel-filtro. São Paulo, Escola Politécnica da Universidade de São Paulo, 2007. 111p. (Tese de Mestrado)

HINES, W.W.; MONTGOMERY, D.C.; GOLDSMAN, D.M. \& BORROR, C.M. Probabilidade e estatística na engenharia. 4.ed. Rio de Janeiro, LTC, 2006. 588p.

LEONG, E.C.; HE, L. \& RAHARDJO, H. Factors affecting the filter paper method for total and matric suction measurements. J. Geotech. Test., 25:322-333, 2002.

MACHADO, J.L.; TORMENA, C.A.; FIDALSKI, J. \& SCAPIM, C.A. Inter-relações entre as propriedades físicas e os coeficientes da curva de retenção da água de um latossolo sob diferentes sistemas de uso. R. Bras. Ci. Solo, 32:495 $502,2008$.

MARINHO, F.A.M. \& OLIVEIRA, O.M. The filter paper method revisited. Geotech. Test. J., 29:1-9, 2006.

MORAES, S.O.; LIBARDI, P.L.; REICHARDT, K. \& BACCHI, O.O.S. Heterogeneidade dos pontos experimentais de curvas de retenção da água no solo. Sci. Agric., 50:393403, 1993.
NASCIMENTO, P.S.; BASSOI, L.H.; PAZ, V.P.S.; VAZ, C.M.P.; NAIME, J.M. \& MANIERI, J.M. Estudo comparativo de métodos para a determinação da curva de retenção de água no solo. Irriga, 15:193-207, 2010.

PEREIRA, E.M. Estudo do comportamento à expansão de materiais sedimentares da formação Guabirotuba em ensaios com sucção controlada. São Carlos, Universidade de São Paulo, 2004. 253p. (Tese de Doutorado)

PIMENTEL-GOMES, F. \& GARCIA, C.H. Estatística aplicada a experimentos agronômicos e florestais. Piracicaba, FEALQ, 2002. 309p.

SOTO, M.A.A. Comparação entre métodos de imposição e de controle de sucção em ensaios com solos não saturados. São Carlos, Universidade de São Paulo, 2004. 224p. (Tese de Doutorado)

TAVARES, M.H.F.; FELICIANO, J.J.S. \& VAZ, C.M.P. Análise comparativa de métodos para determinação da curva de retenção de água em solos. Irriga, 13:517-524, 2008.

TORMENA, C.A. \& SILVA, A.P. Incorporação da densidade no ajuste de dois modelos à curva de retenção de água no solo. R. Bras. Ci. Solo, 26:305-314, 2002.

van GENUCHTEN, M.T. A closed-form equation for predicting the hydraulic conductivity of unsaturated soils. Soil Sci. Soc. Am. J., 44:892-898, 1980.

van GENUCHTEN, M.T.; SIMUNEK, J.; LEIJ, F.J. \& SEJNA, M. RETC version 6.02. Disponível em: <http://www.pcprogress.com/en/Default.aspx?retc-downloads $>$. Acesso em 4 out. 2009. 
